\title{
A thermal neutron scattering law for yttrium hydride
}

\author{
Michael Zerkle ${ }^{\mathrm{a}}$ and Jesse Holmes \\ Bettis Atomic Power Laboratory, Reactor Technology Department, West Mifflin, PA, USA
}

\begin{abstract}
Yttrium hydride $\left(\mathrm{YH}_{2}\right)$ is of interest as a high temperature moderator material because of its superior ability to retain hydrogen at elevated temperatures. Thermal neutron scattering laws for hydrogen bound in yttrium hydride $\left(\mathrm{H}-\mathrm{YH}_{2}\right)$ and yttrium bound in yttrium hydride $\left(\mathrm{Y}^{-} \mathrm{YH}_{2}\right)$ prepared using the $a b$ initio approach are presented. Density functional theory, incorporating the generalized gradient approximation (GGA) for the exchange-correlation energy, is used to simulate the face-centered cubic structure of $\mathrm{YH}_{2}$ and calculate the interatomic Hellmann-Feynman forces for a $2 \times 2 \times 2$ supercell containing 96 atoms. Lattice dynamics calculations using PHONON are then used to determine the phonon dispersion relations and density of states. The calculated phonon density of states for $\mathrm{H}$ and $\mathrm{Y}$ in $\mathrm{YH}_{2}$ are used to prepare $\mathrm{H}-\mathrm{YH}_{2}$ and $\mathrm{Y}$ $\mathrm{YH}_{2}$ thermal scattering laws using the LEAPR module of NJOY2012. Analysis of the resulting integral and differential scattering cross sections demonstrates adequate resolution of the $\mathrm{S}(\alpha, \beta)$ function. Comparison of experimental lattice constant, heat capacity, inelastic neutron scattering spectra and total scattering cross section measurements to calculated values are used to validate the thermal scattering laws.
\end{abstract}

\section{Introduction}

Yttrium Hydride $\left(\mathrm{YH}_{2}\right)$ is of interest as a high temperature moderator material because of its superior ability to retain hydrogen at elevated temperatures. Keinert [1] has proposed a thermal scattering law (TSL) for hydrogen bound in yttrium hydride $\left(\mathrm{H}-\mathrm{YH}_{2}\right)$ based on simple analytic frequency distributions of the Debye- and Gaussian-type. In this paper we extend our prior work [2] to develop TSLs for $\mathrm{H}-\mathrm{YH}_{2}$ and yttrium bound in yttrium hydride $\left(\mathrm{Y}^{-} \mathrm{YH}_{2}\right)$ using higher precision $a b$ initio calculations. The density functional theory (DFT) code VASP [3-5] is used to simulate the face-centered cubic structure of $\mathrm{YH}_{2}$ and calculate the interatomic Hellman-Feynman forces. Lattice dynamics calculations using PHONON [6] are then used to determine the phonon dispersion relations and density of states (DOS). The calculated phonon DOS for $\mathrm{H}$ and $\mathrm{Y}$ in $\mathrm{YH}_{2}$ are used to develop $\mathrm{H}-\mathrm{YH}_{2}$ and $\mathrm{Y}^{-} \mathrm{YH}_{2}$ TSLs using the LEAPR module of NJOY2012 [7]. The TSLs are then validated via comparisons to experimental lattice constant, heat capacity, inelastic neutron scattering spectra, and total scattering cross section measurements.

\section{Structure optimization}

$\mathrm{YH}_{2}$ has a $\mathrm{CaF}_{2}$ type face-centered cubic (FCC) structure. The unit cell is composed of 12 atoms with the hydrogen atoms located in the tetrahedral holes between the $\mathrm{Y}$ atoms. The structure of the $\mathrm{YH}_{2}$ unit cell is shown in Fig. 1 with the $\mathrm{Y}$ atoms colored blue and the $\mathrm{H}$ atoms colored light grey.

The VASP code was used to perform first principles quantum mechanics simulations of the $\mathrm{YH}_{2}$ lattice structure using DFT. The $\mathrm{YH}_{2}$ lattice structure was

a e-mail: michael.zerkle@unnpp.gov optimized using a total electronic energy threshold of $10^{-6} \mathrm{eV}$, a $0.11 \quad 1 / \AA$ k-point spacing $(19 \times 19 \times 19$ k-point mesh), a $500 \mathrm{eV}$ planewave cutoff energy, and a pseudopotential based on the generalized gradient approximation (GGA) of Perdew, Becke and Ernzerhof (PBE) [8] for the exchange-energy correlation. The VASP optimized $0 \mathrm{~K}$ lattice constant of $a=5.2032 \AA$ is in excellent agreement with the experimental value of $5.2032 \pm 0.0003 \AA$ determined by Daou and Vajda [9] using X-ray diffraction measurements at $90 \mathrm{~K}$.

\section{Lattice dynamics calculations}

Lattice dynamics calculations were used to determine the dispersion relations and phonon DOS for $\mathrm{YH}_{2}$ using the PHONON code. Interatomic Hellman-Feynman forces on a $2 \times 2 \times 2$ supercell (96 atoms) with $\pm 0.02 \AA$ asymmetric atom displacements were determined from a series of VASP calculations using the GGA-PBE pseudopotential, a $500 \mathrm{eV}$ planewave cutoff energy, and a $0.0861 / \AA \AA$ k-point spacing $(7 \times 7 \times 7 \mathrm{k}$-point mesh $)$ for the phonon supercell calculations and a $0.111 / \AA \AA$ k-point spacing $(19 \times 19 \times 19 \mathrm{k}$-point mesh) for the electronic energy and DOS contributions to thermodynamic function calculations.

Figure 2 shows the dispersion relations along the highest symmetry points of the Brillouin zone derived from the PHONON calculation. The lower branches are acoustical modes which are mainly due to the heavy $\mathrm{Y}$ atom vibrations. The higher branches are optical modes mainly due to the $\mathrm{H}$ atom vibrations.

Figure 3 shows the partial phonon frequency distributions calculated for $\mathrm{YH}_{2}$. The phonon density of states has two well-separated regions (due to the large mass ratio between $\mathrm{Y}$ and $\mathrm{H}$ ): an acoustical region $(0-0.028 \mathrm{eV})$ which is the preferred region for $\mathrm{Y}$ atom

(C) The Authors, published by EDP Sciences. This is an Open Access article distributed under the terms of the Creative Commons Attribution License 4.0 (http://creativecommons.org/licenses/by/4.0/). 


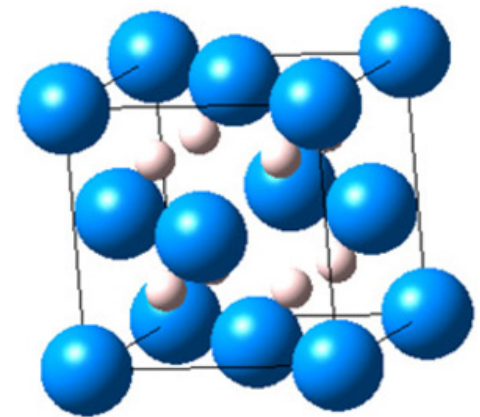

Figure 1. The YH2 unit cell.

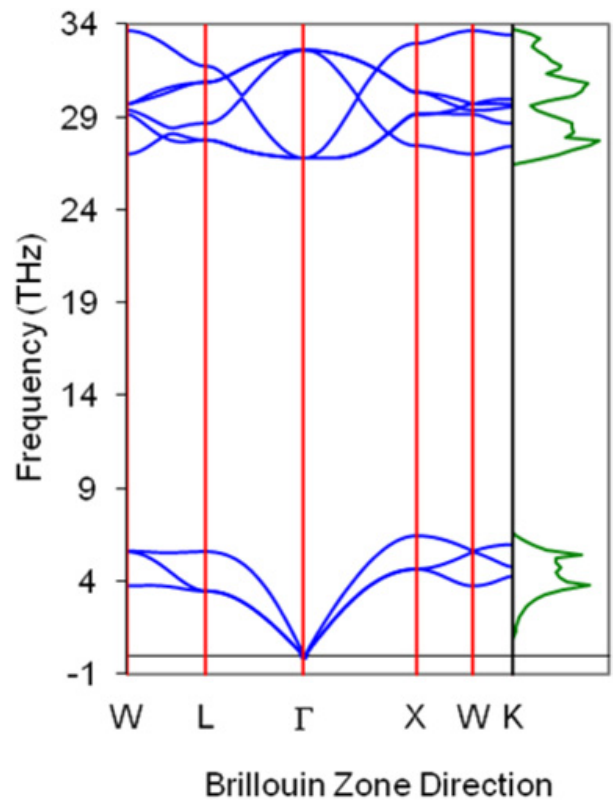

Figure 2. Calculated $\mathrm{YH}_{2}$ dispersion relations.

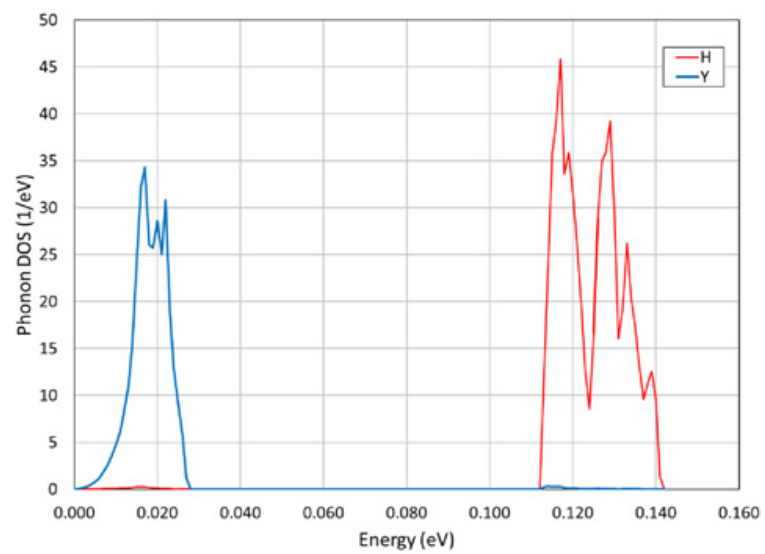

Figure 3. Calculated phonon DOS for $\mathrm{YH}_{2}$.

vibrations and an optical region (0.112-0.142 eV) which is the preferred region for $\mathrm{H}$ atom vibrations. The optical region is centered at $\sim 0.127 \mathrm{eV}$ which compares well with the $0.128 \pm 0.004 \mathrm{eV}$ estimate by Flotow [10] derived from heat capacity measurements and the $0.127 \pm 0.007 \mathrm{eV}$ value recommended by Rush [11] based on inelastic neutron scattering measurements. As shown in Fig. 4, the $\mathrm{YH}_{2}$ optical mode in isotope-dilution neutron scattering measurements by Udovic [12] displayed a similar bimodal structure centered at $\sim 0.127 \mathrm{eV}$.

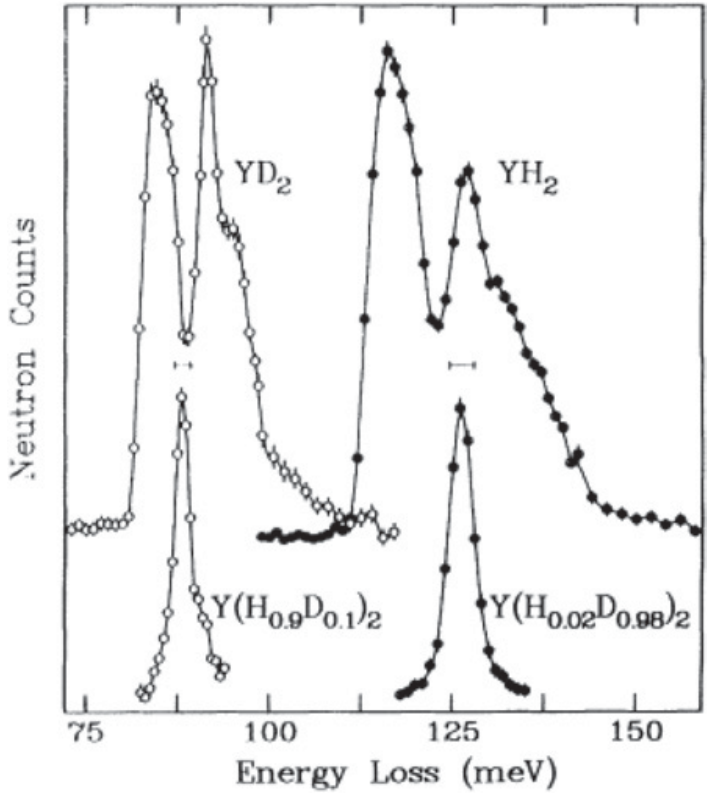

Figure 4. High resolution vibrational spectra of $\mathrm{Y}\left(\mathrm{H}_{x} \mathrm{D}_{1-x}\right)_{2}$ for various values of $x$ from [12].

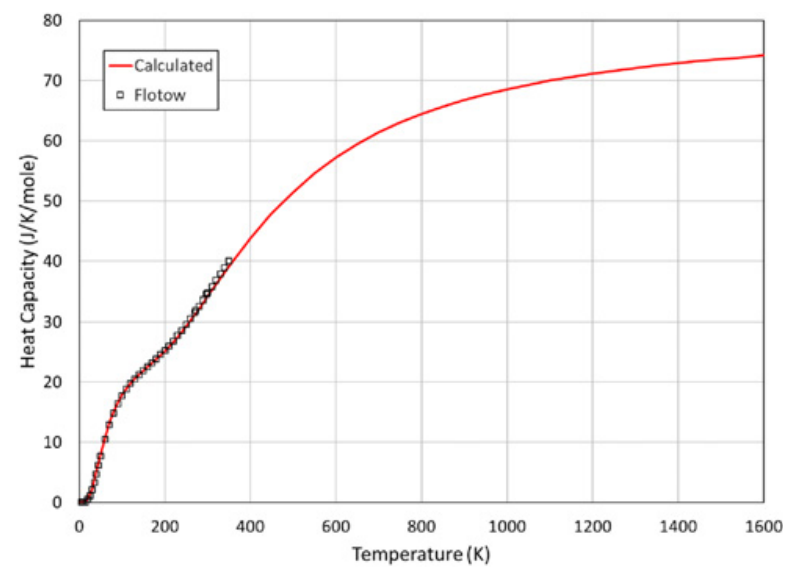

Figure 5. Calculated molar heat capacity for $\mathrm{YH}_{2}$.

Thermodynamic properties of $\mathrm{YH}_{2}$ can also be calculated from the phonon density of states. Fig. 5 shows the calculated heat capacity for $\mathrm{YH}_{2}$ is in agreement with the measurements by Flotow [10].

\section{Thermal scattering laws}

TSLs for $\mathrm{H}-\mathrm{YH}_{2}$ and $\mathrm{Y}-\mathrm{YH}_{2}$ were developed from the calculated partial phonon DOS using the LEAPR module of NJOY2012 [7]. ENDF/B-VII.1 atomic mass ratio and free atom scattering cross section data for ${ }^{1} \mathrm{H}$ and ${ }^{89} \mathrm{Y}$ were used to develop the TSLs. Inelastic scattering is treated in the incoherent approximation. The $\alpha$ (unitless momentum transfer) and $\beta$ (unitless energy transfer) grids were optimized to represent thermal neutron scattering effects up to $4 \mathrm{eV}$ for each evaluation. TSLs were generated at 10 temperatures: 293.6, 400, 500, 600, 700, 800, 1000, 1200, 1400 and $1600 \mathrm{~K}$. Figure 6 shows the inelastic scattering and incoherent elastic scattering cross sections for $\mathrm{H}-\mathrm{YH}_{2}$ calculated by the THERMR module of NJOY2012. The inelastic scattering and incoherent elastic scattering cross sections for $\mathrm{Y}_{-} \mathrm{YH}_{2}$ are given in Fig. 7. 

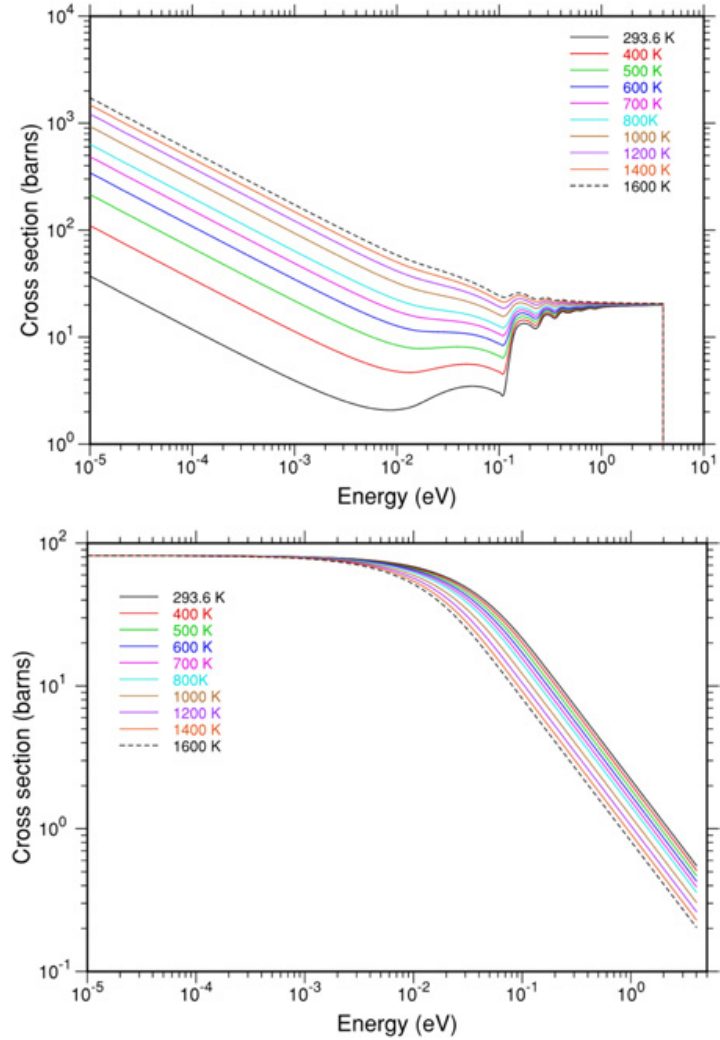

Figure 6. Incoherent inelastic (top) and incoherent elastic (bottom) scattering cross sections for $\mathrm{H}-\mathrm{YH}_{2}$ from THERMR.
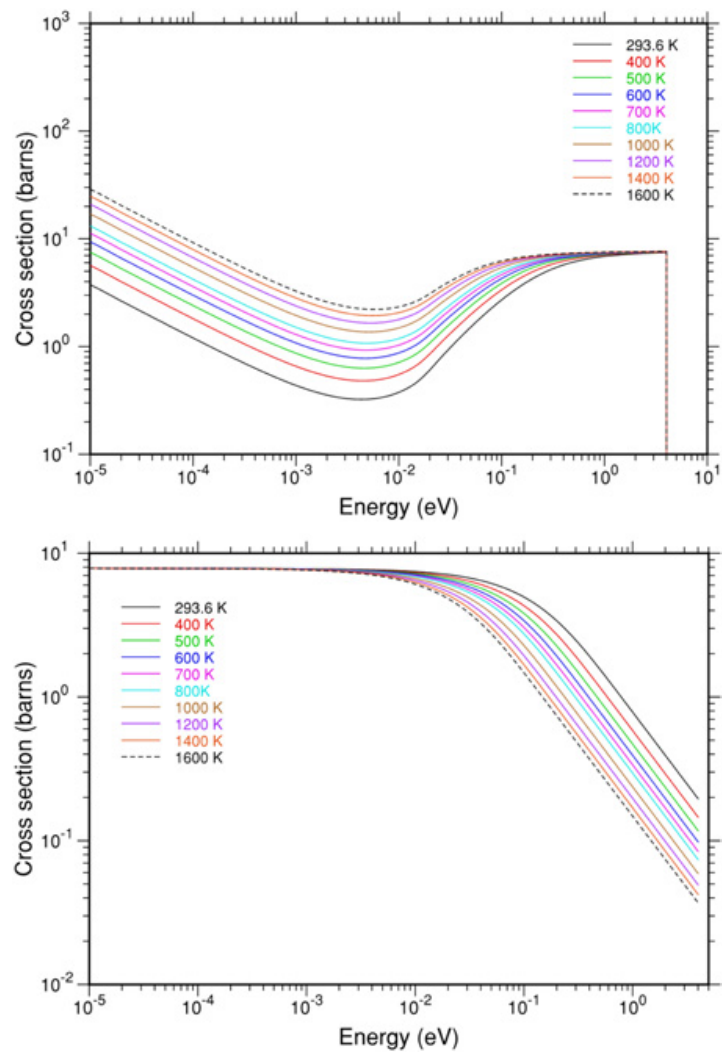

Figure 7. Inelastic scattering (top) and incoherent elastic scattering (bottom) cross sections for $\mathrm{Y}^{-} \mathrm{YH}_{2}$ from THERMR.

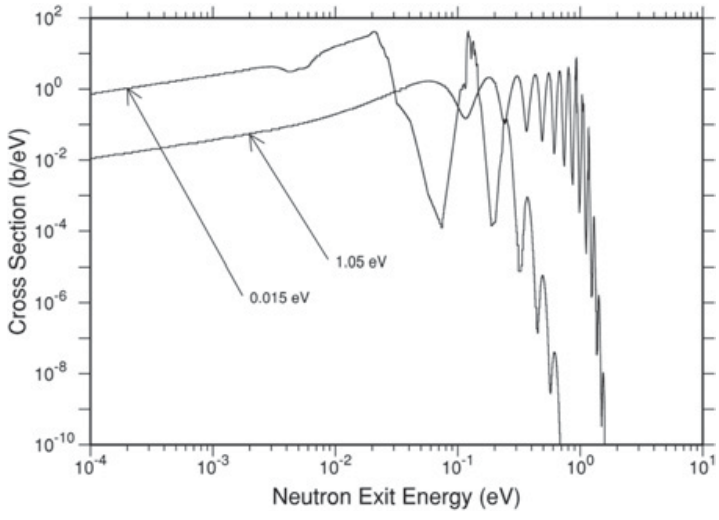

Figure 8. Differential inelastic scattering cross section for $\mathrm{H}-\mathrm{YH}_{2}$ for 0.015 and $1.05 \mathrm{eV}$ incident neutrons.

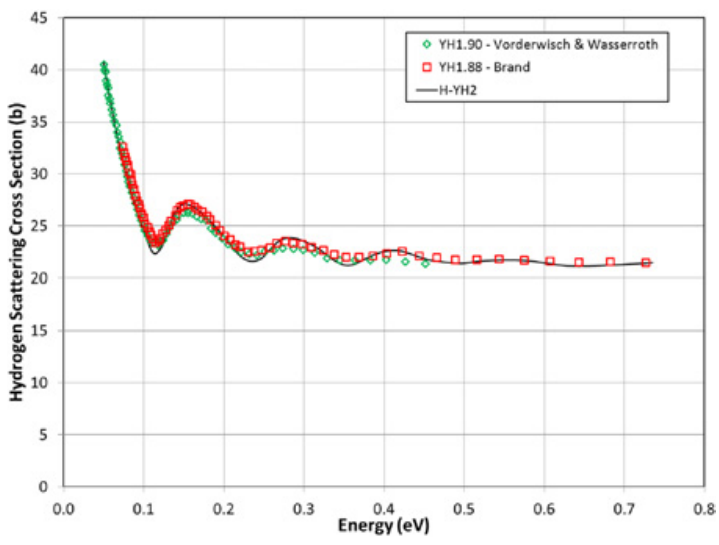

Figure 9. Comparison of the calculated total scattering cross section for $\mathrm{H}-\mathrm{YH}_{2}$ with experimental measurements.

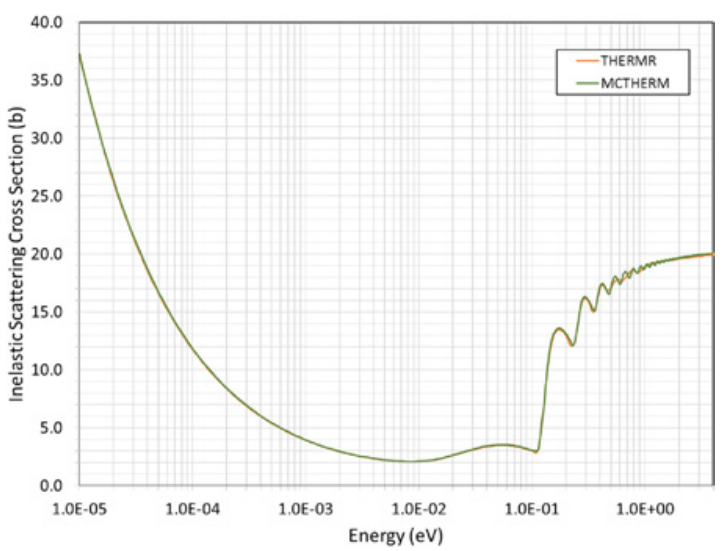

Figure 10. Comparison of the reconstruction of the $\mathrm{H}-\mathrm{YH}_{2}$ inelastic scattering cross section at $293.6 \mathrm{~K}$.

Figure 8 provides the differential inelastic scattering cross section for $\mathrm{H}-\mathrm{YH}_{2}$ at two incident neutron energies. The shape of the acoustic mode and the first optical mode are preserved for $0.015 \mathrm{eV}$ incident neutrons. Above the one-phonon scattering peak, definition in the secondary neutron energy spectrum is lost due to successive convolutions, as expected by theory. For $1.05 \mathrm{eV}$ neutrons, the peak is near $1 \mathrm{eV}$ and the shape of the higher phonon 


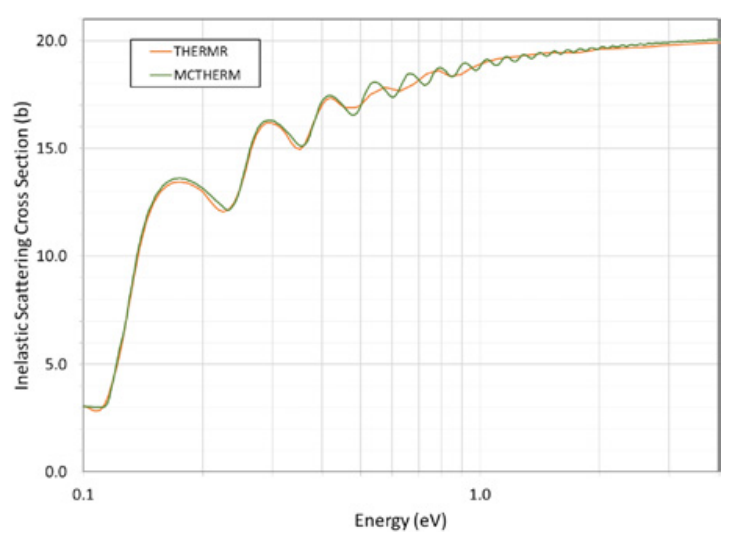

Figure 11. Illustration of inelastic scattering cross section processing issue for $\mathrm{H}-\mathrm{YH}_{2}$ at $293.6 \mathrm{~K}$.

scattering peaks lose definition for both upscattering and downscattering. These results indicate that the $\beta$ grid is sufficiently refined and can accurately capture one-phonon scattering effects.

As shown in Fig. 9, the calculated total scattering cross section for $\mathrm{H}-\mathrm{YH}_{2}$ at room temperature is in good agreement with the $\mathrm{H}-\mathrm{YH}_{x}$ scattering cross section derived from neutron transmission measurements of $\mathrm{YH}_{1.90}$ by Vorderwisch and Waßerroth [13] and $\mathrm{YH}_{1.88}$ by Brand [14].

In Fig. 10 we compare the processing of the inelastic scattering cross section for $\mathrm{H}-\mathrm{YH}_{2}$ at $293.6 \mathrm{~K}$ by THERMR and MCTHERM. MCTHERM is a research code that calculates the inelastic scattering cross section using an ultra-fine energy, $\alpha$, and $\beta$ grid to provide a reference solution. THERMR accurately reconstruct the low-energy region and the first few optical peaks. As shown in Fig. 11, above the third optical peak the automated energy meshing algorithm in THERMR begins smoothing the inelastic scattering cross section and the peaks due to higher phonon order scattering cannot be resolved, but the scattering cross section eventually converges to the free atom scattering cross section. MCTHERM resolves all the optical peaks and captures the actual physical behavior. This processing issue is expected to be present for all hydrogen bound in metal hydride TSLs processed by THERMR. We believe additional user control of the energy mesh refinement algorithm in THERMR is needed to more accurately resolve the physics of hydrogen chemical binding in metal hydrides.

\section{Conclusion}

Thermal neutron scattering laws for $\mathrm{H}-\mathrm{YH}_{2}$ and $\mathrm{Y}^{-} \mathrm{YH}_{2}$ are developed using the phonon DOS produced by first principles DFT and lattice dynamics calculations. The TSLs are validated through comparisons of the calculated lattice constant, phonon DOS, heat capacity, and hydrogen scattering cross section to experimental measurements. The calculated $\mathrm{YH}_{2}$ lattice constant of $a=$ $5.2032 \AA$ is in excellent agreement with experimental value of $5.2032 \pm 0.0003 \AA$ obtained in X-ray diffraction measurements by Daou and Vajda [9]. The calculated phonon DOS is in agreement with inelastic scattering measurements by Udovic [12] and the heat capacity for $\mathrm{YH}_{2}$ derived from the calculated phonon DOS is in agreement with measurements by Flotow [10]. The calculated total scattering cross section for $\mathrm{H}-\mathrm{YH}_{2}$ at room temperature is in agreement with measurements by Vorderwisch \& Waßerroth [13] and Brand [14].

\section{References}

[1] J. Keinert, Atomkernenergie 18, 261-266 (1971)

[2] M. Zerkle, Trans. Am. Nucl. Soc. 111, 821-827 (2004)

[3] G. Kresse and J. Furthmüller, Comp. Matl. Sci. 6, 1550 (1996)

[4] G. Kresse and J. Furthmüller, Phys. Rev. B 54, 11169-11186 (1996)

[5] G. Kresse and J. Furthmüller, Vienna Ab-Initio Simulation Package (Institute of Material Physics, Wien, Austria, 2001)

[6] K. Parlinski, Software PHONON (K. Parlinski, Cracow, Poland, 2002)

[7] A. Kahler, LA-UR-12-27079, Los Alamos National Laboratory (2012)

[8] J. Perdew, K. Burke, and M. Ernzerhof, Phys. Rev. Lett. 18, 3865-3868 (1996)

[9] J. Daou and P. Vajda, Phys. Rev. B 45, 10907-10912 (1992)

[10] H. Flotow et al., J. Chem. Phys. 36, 866-872 (1962)

[11] J. Rush et al., J. Chem. Phys. 45, 3817-3825 (1966)

[12] T. Udovic et al., Phys. Rev. B 50, 15739-15743 (1994)

[13] P. Vorderwisch and K. Waßerroth, Atomkernenergie 14, 370 (1969)

[14] K. Brand, Atomkernenergie 17, 113-120 (1971) 